NIST Technical Note 2015

\title{
Users Guide to the NIST Data Alignment Tool V1.0
}

Michael A. Galler

This publication is available free of charge from:

https://doi.org/10.6028/NIST.TN.2015

National Institute of Standards and Technology

U.S. Department of Commerce 
NIST Technical Note 2015

\title{
Users Guide to the NIST Data Alignment Tool V1.0
}

\author{
Michael A. Galler \\ Energy and Environment Division \\ Engineering Laboratory
}

This publication is available free of charge from:

https://doi.org/10.6028/NIST.TN.2015

September 2018

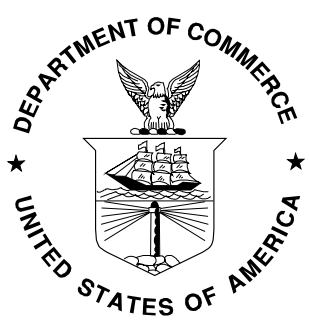

U.S. Department of Commerce

Wilbur L. Ross, Jr., Secretary

National Institute of Standards and Technology 
Certain commercial entities, equipment, or materials may be identified in this document in order to describe an experimental procedure or concept adequately. Such identification is not intended to imply recommendation or endorsement by the National Institute of Standards and Technology, nor is it intended to imply that the entities, materials, or equipment are necessarily the best available for the purpose.

National Institute of Standards and Technology Technical Note 2015 Natl. Inst. Stand. Technol. Tech. Note 2015, 20 pages (September 2018) CODEN: NTNOEF

This publication is available free of charge from: https://doi.org/10.6028/NIST.TN.2015 


\begin{abstract}
The Data Alignment Tool (DAT) was built to assist with the analysis of data used in testing fault detection and diagnostics (FDD) tools and building commissioning (Cx) tools. The data sources were the Virtual Cybernetic Building Testbed (VCBT) [1] and data collected from real buildings. The raw data processed by the DAT typically spanned multiple input files, and was not in a format appropriate for the analysis tools. The features provided by the DAT were implemented to address the specific needs of the tools being tested, but should also be applicable to a wide range of other uses.

The DAT can read data from one or more data files, perform several useful operations on it, and save it with a variety of options. The operations implemented include combining subsets of columns from multiple data files into one output file, reducing data by modifying the time span between data points, performing unit conversions, and filtering data by time of day. Output file operations include a column filter or splitting out files by date. As a manual process, each of these operations would be time consuming, tedious and error prone. The DAT largely automates these operations.
\end{abstract}

\title{
Disclaimers
}

Any mention of commercial products in the DAT or this user's guide is for information purposes only; it does not imply recommendation or endorsement by the National Institute of Standards and Technology (NIST).

This software was developed at NIST by employees of the Federal Government in the course of their official duties. Pursuant to Title 17 Section 105 of the United States Code this software is not subject to copyright protection and is in the public domain. This software is an experimental system. NIST assumes no responsibility whatsoever for its use by other parties, and makes no guarantees, expressed or implied, about its quality, reliability, or any other characteristic.

This software can be redistributed and/or modified freely provided that any derivative works bear some notice that they are derived from it, and any modified versions bear some notice that they have been modified. We would appreciate acknowledgement if the software is used. 


\section{Table of Contents}

1. Introduction to the NIST Data Alignment Tool......................................................... 1

2. Introduction to the DAT User Interface ............................................................................ 3

2.1 Section 1 of the DAT Interface- Options for Reading Data Files ............................. 3

2.2 Section 2 of the DAT Interface — Output Files .................................................... 5

2.3 Section 3 of the DAT Interface- Unit Conversion................................................... 7

2.4 Section 4 of the DAT Interface — Status Display ................................................ 8

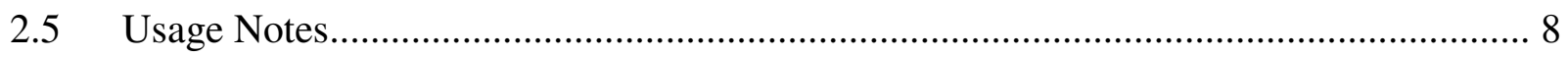

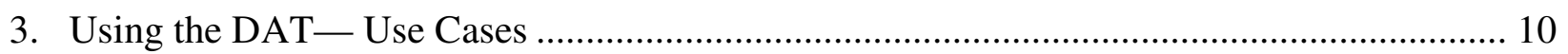

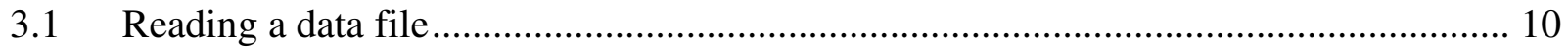

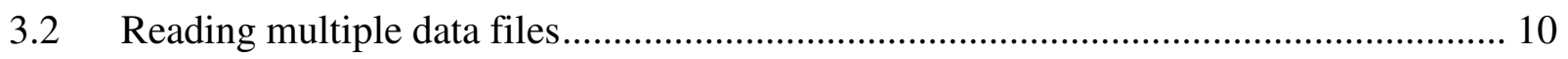

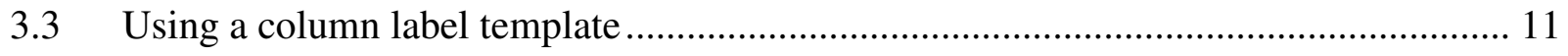

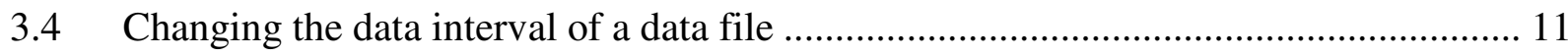

3.5 Reading in a data file with time bracketing..................................................... 12

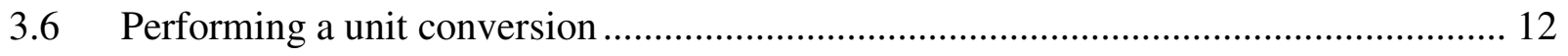

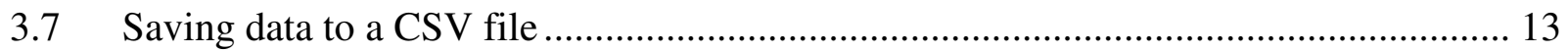

3.8 Saving data to a CSV file with a modified initial date stamp ................................. 13

3.9 Saving data to a CSV file with an output template ............................................. 13

3.10 Saving data to a series of CSV files separated by date ......................................... 14

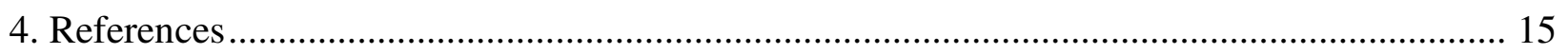




\section{Introduction to the NIST Data Alignment Tool}

The Data Alignment Tool (DAT) was built to assist with the analysis of data used in testing fault detection and diagnostics (FDD) tools and building commissioning ( $\mathrm{Cx}$ ) tools. The data sources were the Virtual Cybernetic Building Testbed (VCBT) [1] and data collected from real buildings using the BACnet Data Source (BDS). The raw data processed by the DAT typically spanned multiple input files, and was not in a format appropriate for the analysis tools. The DAT is built as a 64-bit program and can process large amounts of data. The features provided by the DAT were implemented to address the specific needs of the tools being tested, but should also be applicable to a wide range of other uses.

Each FDD and Cx tool tested had unique requirements relating to the format and order of input data. To analyze the data, specific columns from different series of files often needed to be combined into one file formatted for the specific analysis tool being tested. A large amount of work may be required to prepare the data for analysis for even a single day of testing, and many tests spanned multiple days. As a manual process, this would be time consuming, tedious and error prone. The DAT largely automates this process.

The DAT can read data from one or more data files, perform several useful operations on it, and save it with a variety of options. The operations implemented include combining subsets of columns from multiple data files into one output file, reducing data by modifying the time span between data points, performing unit conversions, and filtering data by time of day. Output file operations include a column filter or splitting out files by date. The columns in the output file may be in any order, and are not restricted by their placement or order in the input files. The column labels are case sensitive and do not use wildcards. This is illustrated in Figure 1, which shows selected data from two files (top) being combined into one file (bottom). The Graphical User Interface for the DAT is shown in Figure 2.

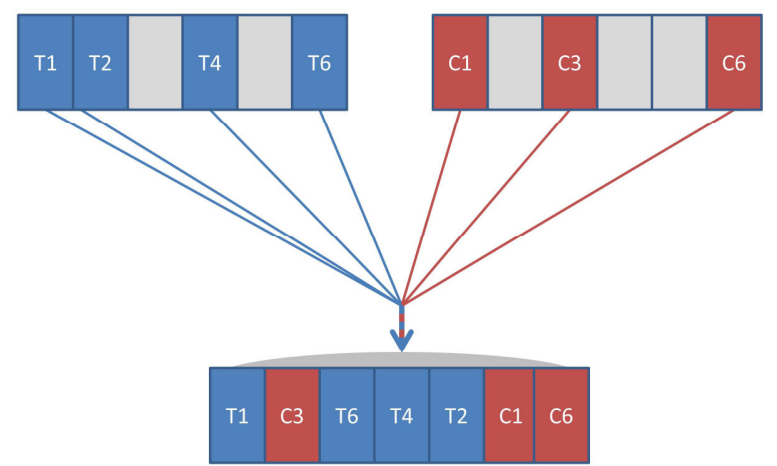

Figure 1: Combining data columns from multiple files

The DAT allows the user to make modifications to the time and date of data input, and to convert the units of some types of data. The time and date modifications involve restricting the time stamp of input data used (bracketing), and modifying the timestamp of the data to start at a 
different time when written to an output file. The DAT also allows the user to optionally fill in missing data. When a row of data is partially filled, the data from the previous timestamp will be copied for the missing data. Data may be saved in files in CSV format or in extensible markup language (XML). These options are explained in more detail in Section 2.

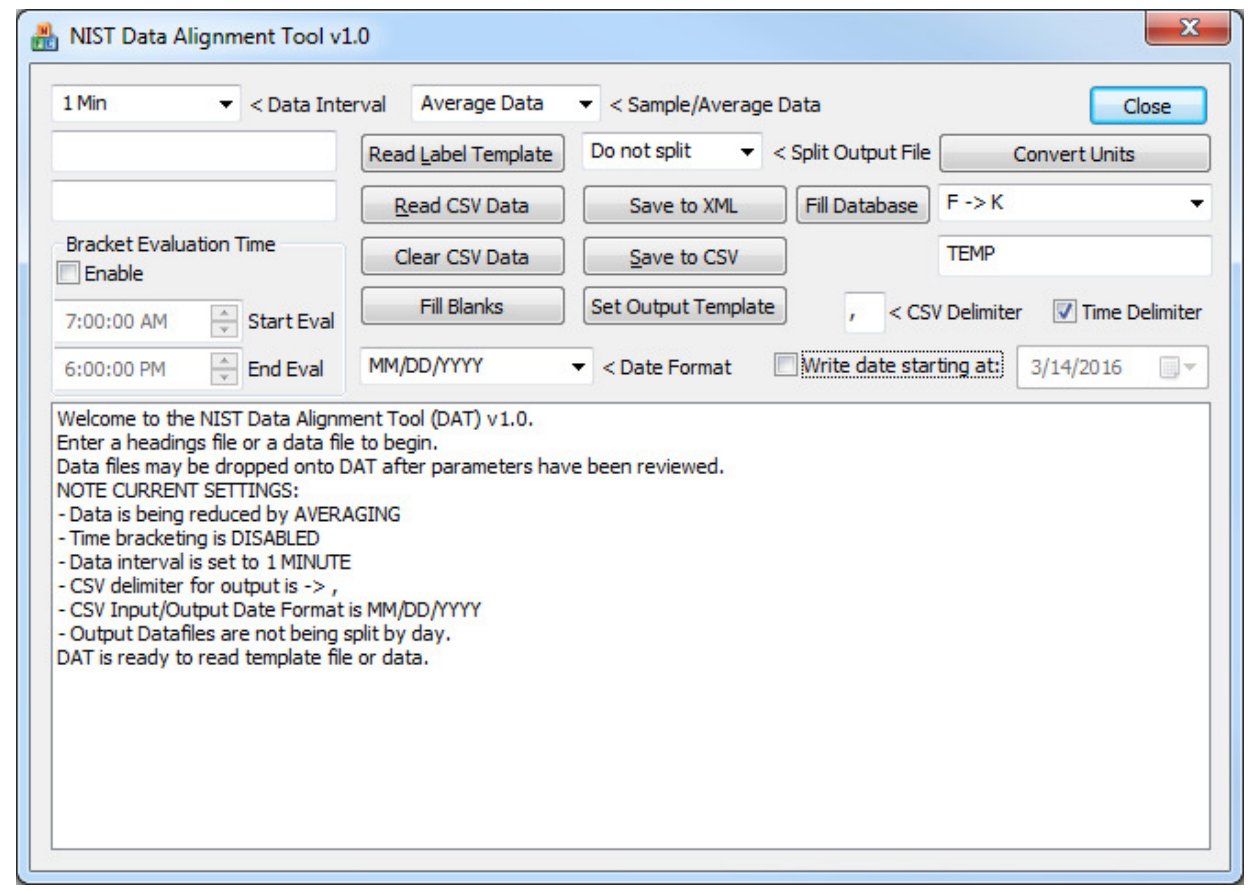

Figure 2: The NIST Data Alignment Tool at startup. 


\section{Introduction to the DAT User Interface}

Interaction with the DAT occurs through the graphical user interface (GUI). The GUI contains four main sections, each with a separate purpose. The sections are enumerated in Figure 3.

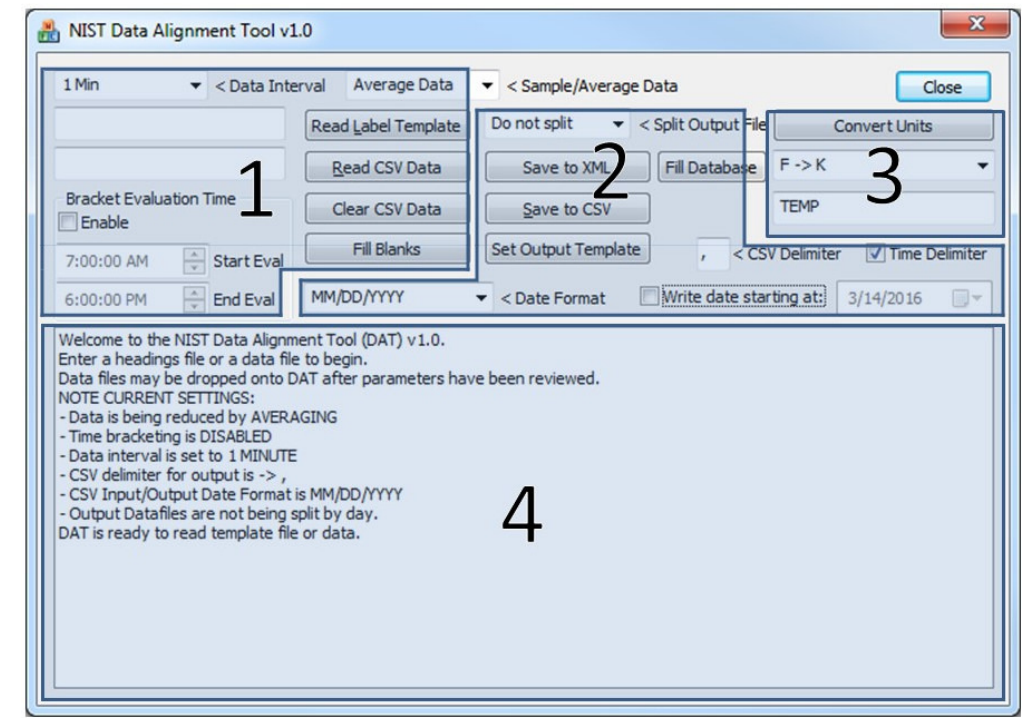

Figure 3: The sections of interest on the DAT GUI.

Each section contains features with related functionality. Section 1 contains controls and options relating to input of data, including methods that allow the user to read data files, to enable bracketing of the analysis time, to set the bracketing start and stop times, and to set the data interval. Section 2 contains controls relating to output of data, including methods that allow the user to save processed data to a new file, adjust the output date stamp, and to specify the separator used in the output files. Section 3 allows the user to convert data that has been read into different units. Section 4 displays status and progress information. These features are described in the following sections.

\subsection{Section 1 of the DAT Interface- Options for Reading Data Files}

Section 1 contains controls relating to input of data, including methods that allow the user to enter configuration files, to read data files, to enable bracketing of the analysis time, and to set the daily start and stop times. This area of the DAT is shown in Figure 4. Each of the controls in Section 1 are discussed below. Use of some of the controls is demonstrated in use cases 1 to 5 in Section 3.

Bracket Evaluation Time: The controls in this section enable the user to bracket the times of day of the data read by the DAT, for example during the occupied period. Times outside of the range entered are discarded when the input data file is read. The bracketing times are applied to each input day equally. Bracketing is enabled by selecting the check box labeled "Enable." If bracketing is to be used, it must be selected before the data files are read. Changes to this setting 
will not be applied retroactively. The bracketing settings may be modified each time a data file is read. Template files are not affected by bracketing.

Data Interval: The data interval determines the span of time between datapoints. Input data with a higher frequency will be reduced to the chosen interval, either by averaging or sampling as selected. Input data with a lower frequency (i.e., undersampled data) will result in missing datapoints when output. The desired data interval is selected by using the pull-down menu next to the label "Data Interval." The available intervals are $10 \mathrm{~s}, 1 \mathrm{~min}, 2 \mathrm{~min}, 5 \mathrm{~min}, 10 \mathrm{~min}, 15$ min, or no interval. The resulting time stamps will start at an increment of the next larger unit, i.e., for the $10 \mathrm{~s}$ interval data will be at $10 \mathrm{~s}, 20 \mathrm{~s}, 30 \mathrm{~s}$, etc. For the $5 \mathrm{~min}$ interval data will be at $5 \mathrm{~min}, 10 \mathrm{~min}, 15 \mathrm{~min}$ etc. The no interval option allows the data to be read without modification. If the interval of the data read matches the selected interval, the data will also not be modified.

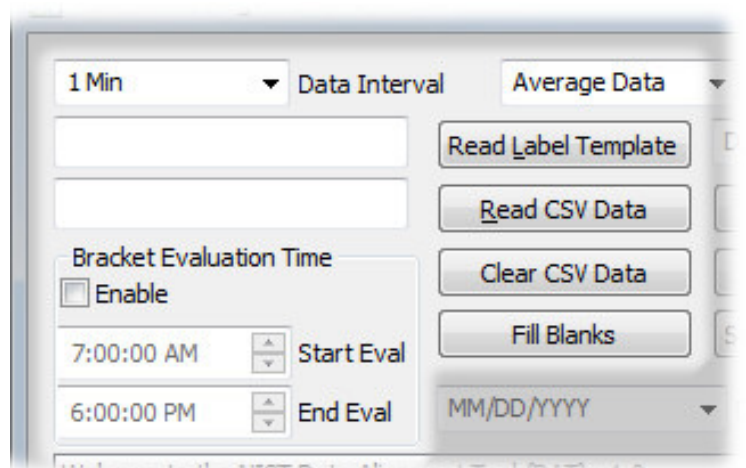

Figure 4: Section 1 of the DAT: options for reading data files.

Sample Data or Average Data: The pull-down menu labeled "Sample/Average Data" allows the user to determine the method used to reduce the data. If the Average Data option is selected, the data will be averaged over the selected Data Interval. If the Sample Data option is selected, the data point closest to each time indicated by the Data Interval will be used, and the remainder of the data will be discarded.

Column Label Template: The column label template is read by pressing the button labeled "Read Label Template" and selecting the correct file in the file selection window. The column label template tells the DAT the labels of the columns in the output file and their order. The template file is a CSV file with the first line consisting of the labels. Further lines in the file are ignored. A previously configured data file may be used as the label template. If no such data files are available, as may be the case the first time the DAT is used, a template file may be assembled manually. If no column label template is entered, the columns in the first data file read will be used as the template. 
Read CSV Data: Data is read by pressing the button labeled "Read CSV Data" and then selecting one or more CSV files in the file selection window. An XML file previously saved by the DAT may also be read. If a column label template has not been selected, the columns in the first data file read will be used as the template. Note that if multiple files are to be read, the XML file should be read first. If there are multiple files to be read, they may all be selected to be read at one time, or they may be read one at a time by multiple presses of the "Read CSV Data" button.

Clear CSV Data: Data that has been read may be cleared from storage by pressing the "Clear CSV Data" button. This will only erase the data stored by the program, and will not affect the original data files or the column label template. Additional data files may then be read.

Fill Blanks: When data is collected from more than one source, it is possible that for some datapoints the timestamps will fail to match, resulting in timestamps that do not receive data from all input sources. This results in blank positions in the data, which may cause errors or improper analysis when read by some analysis tools. Using the "Fill Blanks" button causes the DAT to search for these blank positions and fill them with the value from the preceding timestamp. After this operation, the total number of blanks that have been filled is displayed to the user. An excessive number of blank positions may indicate a problem with the input data or settings.

\subsection{Section 2 of the DAT Interface- Output Files}

The controls in this section allow the user to save the data to a CSV file or XML file, or to a series of files. The user may select an output template, which works similarly to the input template, and specify the delimiter used in the output CSV files. The user may also modify the date stamp on the output data, which is useful if the date recorded with the data is incorrect. The controls described in this section are shown in Figure 5. Each of the controls in Section 2 are discussed below. Use of some of the controls is demonstrated in use cases 7 to 10 in Section 3.

Save to XML: The Save to XML option can be used to save data sets between runs of the DAT. The XML file also contains metadata about the column template and the data files that have been read, including the file name, path, and the order of columns. The file is saved in the XML format that the DAT uses internally to store data, and may be reentered when restarting the DAT.

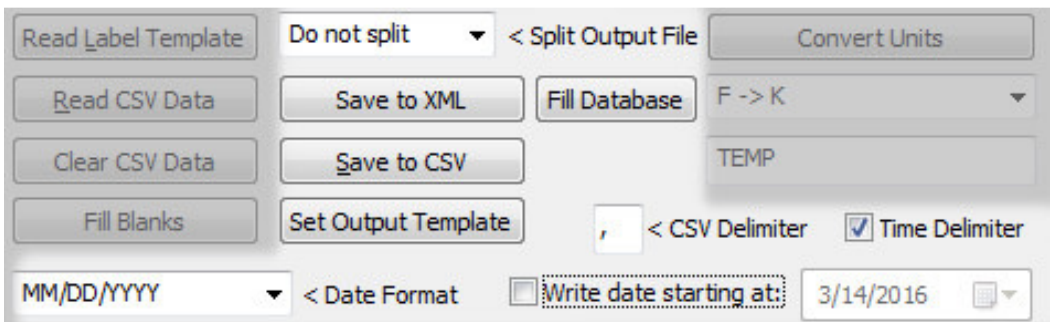

Figure 5: Section 2 of the DAT: save options for output files. 
Save to CSV: The Save to CSV option is used to export data to a CSV file. The output options should be reviewed before saving, and are explained below. The CSV file will contain a header line consisting of the labels for each column, followed by lines containing data. The data lines start with a date stamp, followed by the data. The date will be in the format specified in the Date Format box, which is explained below. The exported CSV file can be opened using a spreadsheet program, a text editor, or any other application which can read CSV files. The exported CSV file may also be read by the DAT in a future session to further modify the data.

Split Output File: Output CSV files may be split by day, which creates a series of output files. If this option is chosen, file lengths of 1 to 7 days may be selected. The files will be split with the data in each file starting at the first data point after midnight, regardless of the starting time of the data. If a length of more than one day is chosen, the first day will be included even if it contains less than a full day of data. The output files will be indicated by appending an 'S\#\#' to the file name mask, i.e., if the output file name mask is "MyData", the output files would be named "MyData S01.csv", "MyData S02.csv" and so on until all data had been saved.

Set Output Template: Subsets of columns may be designated for output in CSV files by using an output template. An output template is selected by clicking on the 'Set Output Template' button, which opens a file selection dialog box. The output template functions similarly to the input template. The user is given the choice to delete previously entered output templates when selecting a new one.

CSV Delimiter: The control labelled "CSV Delimiter" allows the user to specify the delimiter used in CSV files. This is useful because some applications use different symbols as the delimiter in CSV files, despite the name of the format.

Time Delimiter: The control labelled "Time Delimiter" will cause the program to use the CSV delimiter between the date and time fields in the output CSV file. It is selected by default. If this box is not checked there will be no delimiter between the date and time fields.

Write Date Starting At: To enable changing the initial date stamp in the output data, select the checkbox labeled "Write date starting at:" as shown in Figure 5 and then use the standard date picker to select the initial date stamp. This feature is intended to correct date stamps when they do not correspond to the actual date the data represents, i.e., when the date on the data acquisition system is incorrect. This can happen with a hardware in the loop simulation when the simulation time does not match the clock time of the data acquisition system. The time of day associated with each datapoint will not be changed. If the data covers multiple days, the date written will increment appropriately. Note that using this feature will change the date stamp for all data, and does not select a range of data to save. For example, if a date corresponding to the second day of data is selected, the full data set will be saved but with the date stamp of each datapoint modified to be two days later. 
Date Format: The output date format may also be modified. The output date format will be written in the format specified in the drop-down menu labelled "Date Format." Note that this is the same control used to specify the input date format.

\subsection{Section 3 of the DAT Interface- Unit Conversion}

The controls in this section allow the user to convert the units of columns of data that have been read. The available conversions are listed in Table 1 . To perform a conversion, select one of the conversions from the drop-down menu. The conversions are listed in (from) $\rightarrow$ (to) order, where the current units are (from) and the desired units are (to).

Table 1: Available Unit Conversions.

\begin{tabular}{|cccc|}
\hline Conversion \# & From & To & Default Label \\
\hline 1 & ${ }^{\circ} \mathrm{F}$ & $\mathrm{K}$ & TEMP \\
\hline 2 & $\mathrm{CFM}$ & $\mathrm{Kg} / \mathrm{s}^{\text {a }}$ & FLOW \\
\hline 3 & ${ }^{\circ} \mathrm{F}$ & ${ }^{\circ} \mathrm{C}$ & TEMP \\
\hline 4 & $\mathrm{CFM}$ & $\mathrm{L} / \mathrm{s}^{\mathrm{a}}$ & FLOW \\
\hline 5 & $\mathrm{~K}$ & ${ }^{\circ} \mathrm{C}$ & TEMP \\
\hline 6 & $\mathrm{Kg} / \mathrm{s}$ & $\mathrm{L} / \mathrm{s}^{\mathrm{a}}$ & FLOW \\
\hline 7 & Percent & Fraction & Valve \\
\hline
\end{tabular}

${ }^{\text {a }}$ Conversion uses dry air at $20{ }^{\circ} \mathrm{C}$, with a density of $1.2 \mathrm{~kg} / \mathrm{m}^{3}$.

When a conversion is selected, a default search word is entered in the text box below the dropdown menu. This is shown as the text "TEMP" in Figure 6. The user may edit the default search text in the box to match their column headings. The search text will be searched for in column headings, and columns with matching text will be converted. If the column label contains the

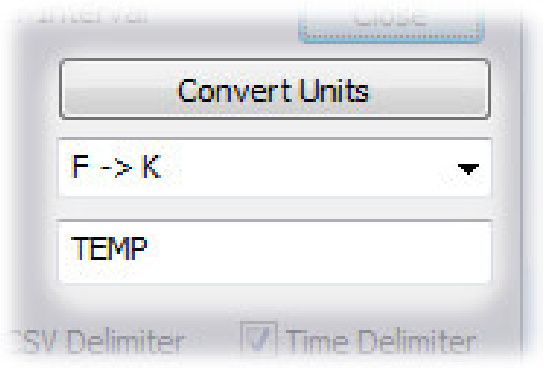

Figure 6: Section 3 of the DAT: unit conversions.

search text as a portion of its text, the selected conversion will be applied to that column. The selection is case sensitive. For example, if the search text is "Temp", then all columns with labels such as "SATemp", "RA_Temp" or "Temp-OA" would be selected. Columns with labels such as "SA-temp", "MA_TEMP" or "OARH" would not be selected. Use of the unit conversion functionality is demonstrated in use case 6 in Section 3. 
The methods for the temperature conversions are straightforward. Conversion 7 is used to convert numbers from the range 0 to 100 to 0 to 1 . For Conversions 2 and 6 , which are flow rate conversions, the fluid is assumed to be air with a density of $1.2 \mathrm{~kg} / \mathrm{m}^{3}$, which is the density of dry air at $20^{\circ} \mathrm{C}$. If the data to be converted do not match these conditions, another method should be used.

\subsection{Section 4 of the DAT Interface - Status Display}

The controls in this section display information about the status of operations initiated by the user, such as reading files, converting units, saving files, or other operations selected by the user. The text shown will vary depending on the exact operations chosen by the user. The status screen at startup is shown in Figure 7.

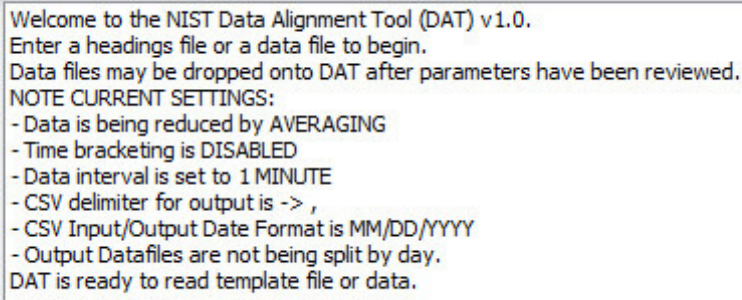

Figure 7: Section 4 of the DAT: status display.

\subsection{Usage Notes}

This section contains additional information about usage of the DAT.

Time and date: The date and time must be at the beginning of each data line in input CSV data files. They may be in one or two columns, depending on the selected format. The date must be formatted as shown in the Date Format box, by default MM/DD/YYYY, and the time as HH:MM:SS, where seconds are optional. For example, 4:00 PM on March 14, 2016 would be written as 03/14/2016, 16:00:00. Other examples for this time are given in Table 2. Time/date stamp formats 1 to 5 have the date in the first column, and time in the second column. Time/date stamp formats 6 to 8 have the time and date in the first column, and data starting in the second column. Time/date stamps 9 and 10 have two complete time/date stamps. Time/date stamp formats 11 and 12 follow ISO 8601 extended and basic formats for calendar dates. Note that for time stamp formats 6 to 12 the length of each field must be as specified.

After the date and time columns in an input CSV file, any column labels where the label contains the strings 'DATE' or 'TIME' will be ignored, until a valid column label is found. After a valid column label is found, these strings are not checked for. Any label without those strings and consisting of ASCII characters 32 to 126 is considered valid. This check is necessary to process 
some data files generated using the BACnet Data Source (BDS) [2] and BACnet Communications DLL [3] while collecting data from the VCBT. The BDS applied two sets of time/date stamps to these files, one set for the actual time, and one set for the time in the HVAC controllers and computer simulations. The controller/simulation time was used for aligning data, while the real time/date stamp was saved to verify that data was collected properly. Time formats 9 and 10 in Table 2 should be used when dual time/date stamps are present. Time format 9 will retain the first time/date stamp, and time format 10 will retain the second time/date stamp.

If modifying an XML file, the user should follow the format used in the file. Note that XML files are useful as an intermediate step, such as to save progress reading or modifying data, and are unlikely to be useful as final output.

Table 2: Examples of Different Time/Date Stamp Formats, Using March 14, 2016, 4 PM.

\begin{tabular}{|c|c|c|c|}
\hline ID & Format & Column 1 & Column 2 \\
\hline 1 & DD/MM/YYYY & $14 / 3 / 2016$ & 16:00:00 \\
\hline 2 & MM/DD/YYYY & $3 / 14 / 2016$ & 16:00:00 \\
\hline 3 & DD/MM/YY & $14 / 3 / 16$ & 16:00:00 \\
\hline 4 & MM/DD/YY & $3 / 14 / 16$ & 16:00:00 \\
\hline 5 & YYYY/MM/DD & $2016 / 3 / 14$ & 16:00:00 \\
\hline 6 & YYMMDD-hh:mm:ss & 160314-16:00:00 & (data starts) \\
\hline 7 & YYYYMMDD-hh:mm:ss & 20160314-16:00:00 & (data starts) \\
\hline 8 & YYYYMMDD-hhmmss & 20160314-160000 & (data starts) \\
\hline 9 & YYYYMMDD-hhmmss ${ }^{a}$ & $20160314-160000$ & 20160314-155539 \\
\hline 10 & YYYYMMDD-hhmmss ${ }^{\text {b }}$ & 20160314-160000 & 20160314-155539 \\
\hline 11 & YYYY-MM-DDThh:mm:ss & 2016-03-14T16:00:00Z & (data starts) \\
\hline 12 & YYYYMMDDThhmmss & $20160314 \mathrm{~T} 160000 \mathrm{Z}$ & (data starts) \\
\hline
\end{tabular}

a Time format 9 will retain the first time/date stamp

$\mathrm{b}$ Time format 10 will retain the second time/date stamp

Set Output Template: Subsets of columns may be designated for output in CSV files by using an output template. An output template is selected by clicking on the 'Set Output Template' button, which opens a file selection dialog box. Output Templates are identical to Input Templates except for usage, and can be used interchangeably. If the user fails to select an input template before reading data, they may get the same result by using the input template as an output template. The advantage offered by the output template is found when there is one large data file to be divided into multiple files, i.e., if it contains data from multiple HVAC components which will be split into individual files. Using an input template, the data would have to be read once for each desired output file. Using output templates instead, the data would be read one time and then a different output template would be selected for each desired output file. The user is given the choice to delete previously entered output templates when selecting a new one. 


\section{Using the DAT - Use Cases}

This section instructs the user in how to use the DAT through various examples given by use cases. Various interactions a user could initially or commonly have with the DAT are described in the use cases presented here. This is not an exhaustive list, but a set chosen to be sufficient to allow a new user to become familiar with and use the DAT. The use cases described are:

1. Reading a data file.

2. Reading multiple data files.

3. Using a column label template.

4. Changing the data interval of a data file.

5. Reading in a data file with time bracketing.

6. Performing a unit conversion

7. Saving data to a CSV file.

8. Saving data to a CSV file with a modified initial date stamp.

9. Saving data to a CSV file with an output template.

10. Saving data to a series of CSV files separated by date.

\subsection{Reading a data file}

Data may be read by the DAT without first selecting a column label template. In this case, the column labels and order of the first input file will be used as the column label template. To read data files, complete the following activities:

1. Start the DAT. Check that the input options are appropriate.

2. Click on the "Read CSV Data" button.

3. Use the file selection window to select the input data file.

4. Click on the "Open" button. The DAT will read the data file, using the first row as the column label template.

Alternative method:

1. Start the DAT. Check that the input options are appropriate.

2. Select your file in Windows Explorer, drag it over any portion of the DAT window, and release it (i.e., drag and drop). The DAT will read the data file as above.

You may now perform other actions, such as repeating these steps to read additional data files, unit conversions, or saving your data to a new file.

\subsection{Reading multiple data files}

Multiple data files may be read by the DAT at the same time. The files must be located in the same directory. The files do not need to have the same columns. If the files being read have varying column labels, it is recommended to either first read in a single file with the desired final column labels, or to use a column label template before reading multiple files. If reading an XML data file, it should be read before any CSV data files. To read multiple data files, complete the following activities:

1. Start the DAT. Check that the input options are appropriate. 
2. Click on the "Read CSV Data" button.

3. Use the file selection window to select multiple input data files.

4. Click on the "Open" button. The DAT will read the data files, using the first row of the first file read as the column label template.

Alternative method:

1. Start the DAT. Check that the input options are appropriate.

2. Use Windows Explorer to select multiple input data files, drag them over any portion of the DAT window, and release them (i.e., drag and drop). The DAT will read the data files as above. The files many be read singly or in groups.

\subsection{Using a column label template}

The column label template is useful when reading in multiple data files, or when reducing the number of columns read from one file. A previously generated data file in CSV format may be used as the column label template. If such a file is not available, a column label template file may be constructed using a text editor or other program which can save data to CSV format, such as a spreadsheet program. A sample column label template is given in Table 3. Note that an actual column label template would probably use more columns. To use a column label template, complete the following activities:

1. Start the DAT.

2. Press the button in Section 1 labeled "Read Label Template."

3. Use the file selection window to select the column label template file.

4. Click on the "Open" button. The DAT will read the template file, and apply it to all new data files read.

You may now read data files, as described in previous use cases.

Table 3: Sample Column Label Template File in CSV Format.

Supply Temp., Supply Temp. SP, Outdoor Air Temp., Return Air Temp., Mixed Air Temp.

\subsection{Changing the data interval of a data file}

Data read by the DAT may have been collected at a frequency greater than is necessary for analysis. The DAT can reduce the amount of data saved by lowering the frequency of saved datapoints, for example changing the time between datapoints from $10 \mathrm{~s}$ to $1 \mathrm{~min}$. When reducing data, the user has the options of averaging data or sampling data. These options are described in Section 2.1. To change the data interval of a data file, complete the following activities:

1. Start the DAT. 
2. Click on the pull-down menu in Section 1, labeled "Data Interval" and select an interval greater than the one used in the input data file.

3. Select the desired option in the pull-down menu in Section 1 labeled "Sample Data or Average Data Over Interval."

You may now read data files as described in previous use cases, or perform other actions using the DAT.

\subsection{Reading in a data file with time bracketing}

When reading data into the DAT, data with a time of day outside of a user defined span may be excluded. An example of when this would be used is to exclude data from when a building is in unoccupied mode from an analysis. The controls in Section 1 of the DAT GUI interface allow the user to enable time bracketing, and to select a start and stop time. This setting must be enabled before data files are read by the DAT. Time bracketing is applied to data in each day, but does not exclude any data by date. To use the time bracketing feature, complete the following activities.

1. Start the DAT.

2. Select the checkbox in Section 1, under the label "Bracket Evaluation Time" labeled "Enable." The DAT will enable the controls for start and stop time, located immediately below the check box.

3. Set the start time for data analysis in the time selection box next to the label "Start Eval."

4. Set the stop time for data analysis in the time selection box next to the label "End Eval."

You may now read data files as described in previous use cases, or perform other actions using the DAT.

\subsection{Performing a unit conversion}

The DAT can convert values into different units, based on the column label. There are several conversions available, as listed in Table 1. Selection of a column for conversion is based on the label mask and on the column label. If the column label contains the mask as a portion of its text, the selected conversion will be applied to that column. The selection is case sensitive. For example, if the column mask is "Temp", then all columns with labels such as "SATemp," "RA_Temp" or "Temp-OA" would be selected. Columns with labels such as "SA-temp," "MA_TEMP" or "OARH" would not be selected. To use the unit conversion feature, complete the following activities:

1. Start the DAT.

2. Read data as described in previous use cases.

3. Using the drop-down menu under the button in Section 3 labeled "Convert Units" select a conversion that is appropriate for your data.

4. Confirm that the mask is appropriate for the column labels to be converted. Modify the mask if needed.

5. Click on the button labeled "Convert Units." The DAT will search for columns with labels which match the mask, and convert the data in them. 
6. Repeat Steps 2 to 5 as appropriate for other types of data.

It is possible to have data from different sources that use different units, i.e., one system records in temperature units of Celsius, while another uses Fahrenheit. In this case, the above method would not be appropriate as the data that was already in Celsius would undergo the conversion, unless the sources used different labels. A recommended method to process this data would be to first read one set of data, perform the appropriate conversions, and then load the remaining data. In this example, if the desired units are Celsius, then the data file using Fahrenheit units would be read and converted to Celsius units. The remaining data files could then be read.

\subsection{Saving data to a CSV file}

The DAT allows the user to save data to a CSV file. To save data to a CSV file, complete the following activities:

1. Start the DAT.

2. Read data as described in previous use cases and perform any other operations as desired.

3. Select the button in Section 2 labeled "Save to CSV." This will open a file selection dialog box.

4. Use the file selection window to select the data file or enter the name.

5. Click on the "Save" button. The DAT will save the data file.

\subsection{Saving data to a CSV file with a modified initial date stamp}

When saving data to an output file, the user may want to set a specific date for the start of the data. The DAT allows the user to specify an alternate initial date stamp for the data. This may be required if the date is not set correctly on the data acquisition system. The dates in the output data will be incremented to match date changes in the input data. The time stamps of the data will not be changed. To enable the option to modify the initial date stamp, complete the following activities:

1. Start the DAT.

2. Read data as described in previous use cases, and perform any other operations as desired.

3. Select the checkbox in Section 2 next to the label "Write date starting at:" and use the time selection box to select the desired start date.

4. Save the data using methods described in use Section 3.7.

\subsection{Saving data to a CSV file with an output template}

When saving data to a CSV output file, the user may want to save a subset of the columns that were originally entered. An example of this would be when one data file contains data from multiple pieces of HVAC equipment, but the analysis tool is designed to read data for each piece of equipment in a separate file. Note that one output template file will be necessary for each output data file. To use the output template feature, complete the following activities:

1. Start the DAT. 
2. Read data as described in previous use cases, and perform any other operations as desired.

3. Click on the button in Section 2 labeled "Set Output Template." This will open a file selection dialog box.

4. Use the file selection window to select the column label template file.

5. Click on the "Open" button. The DAT will read the template file.

6. Save the data to a CSV file, using any of the other save options listed above.

7. Repeat Steps 2 and 3 as necessary. Note that when reading subsequent output template files the user will be given the option of deleting previous templates.

8. Save the data using methods described in use Section 3.7.

This feature does not work with XML output files.

\subsection{Saving data to a series of CSV files separated by date}

When saving data to an output file, the user may want to save the data into files that are separated by date. This may be to aid with certain types of analysis, or to reduce the size of output files. To use this feature, complete the following activities:

1. Start the DAT.

2. Read data as described in previous use cases, and perform any other operations as desired.

3. Select the pull-down menu in Section 2 next to the label "Split Output File" and select the entry corresponding to the number of days of data wanted in each output file. The lengths available are 1 to 7 days.

4. Save the data using methods described in use Section 3.7. 


\section{References}

[1] Bushby, Steven T., et al., 2009, "The Virtual Cybernetic Building Testbed- A Building Emulator," ASHRAE Transactions, Volume 116, Part 1, p37.

[2] Galler, Michael A., 2011, "Using the BACnet Data Source v1.7", NISTIR 7825.

[3] Galler, Michael A., 2018, "Programmers Guide to the BACnet Communications DLL", NISTIR (pending) 